\title{
Cuidados de enfermagem ao recém-nascido nos distintos cenários: revisão integrativa
}

\author{
Aneís Louise Peres¹, Márcia Helena de Souza², Michelle Thais Migoto³, Gabrielle Freitas ${ }^{4}$
}

\section{RESUMO}

Objetivo: Identificar os cuidados de enfermagem ao recém-nascido. Método: Revisão integrativa com referencial metodológico de Ganong, que utilizou descritores "enfermagem neonatal", "cuidados de enfermagem" e "recémnascidos", intercalados ao operador booleano "and", nas bases de dados Cinahl, Pubmed, Scopus, Web of Science e Medline, entre 2015 e 2017. O critério de inclusão foi mediado pela pergunta norteadora estruturada a partir do acrônimo PICo: Qual é o estado da arte dos cuidados de enfermagem neonatais humanizados realizados nos diferentes cenários da atenção? Após selecionados os artigos $(n=16)$, aplicou-se o instrumento Measuring Study Quality para avaliar a qualidade metodológica dos artigos, através de dupla checagem, decidindo-se pela inclusão de 10 artigos na amostra. Resultados: Das publicações analisadas, 60\% são nacionais, enquanto as internacionais são dos EUA, Holanda, Itália e Indonésia, sendo, em sua maioria (80\%), relativos à atenção aos recém-nascidos prematuros. Discorrem sobre cuidados relacionados a suporte respiratório, prática de sono seguro, manuseio mínimo, redução dos estímulos sensoriais e ambientais, conforto, posicionamento, higiene corporal, pesagem e prevenção de lesões de pele. Conclusão: As publicações concentram-se na produção de conhecimento para a qualificação dos profissionais de Unidade de Terapia Intensiva Neonatal (UTIN), com foco em prematuros. Constatou-se uma lacuna de pesquisas sobre a assistência neonatal na Sala de Parto, Alojamento Conjunto, na Unidade de Cuidado Intermediário Canguru (UCINCa) e na Atenção Primária à Saúde.

Descritores: Recém-nascido; Enfermagem Neonatal; Cuidados de Enfermagem; Revisão; Tecnologia; Enfermagem Baseada em Evidências.

1 Enfermeira. Universidade Federal do Paraná. Curitiba, Paraná, Brasil. aneperes44@gmail.com. ORCID iD: https://orcid.org/0000-0003-2702-3037

2 Enfermeira. Doutora em Saúde Pública. Universidade Federal do Paraná. Docente do Departamento de Enfermagem. Curitiba, Paraná, Brasil.marciahelenafreire@gmail.com ORCID iD: https://orcid.org/0000-0003-47883221

3 Enfermeira. Doutoranda em Enfermagem. Universidade Federal do Paraná. Curitiba, Paraná, Brasil. michellemigoto@gmail.com. ORCID iD: https://orcid.org/0000-0002-8546-8694

4 Enfermeira. Doutoranda em Enfermagem. Universidade Federal do Paraná. Curitiba, Paraná, Brasil. gabisaga@gmail.com https://orcid.org/0000-0001-9716-659X

\section{Autor Correspondente}

Aneís Louise Peres

Endereço: Av. Prefeito Lothário Meissner, 632. Jardim Botânico, Curitiba,

Paraná, Brasil.

Fone: (41) 9 99677433/(41) 32469649

Email: aneperes44@gmail.com.

Data de submissão: $31 / 08 / 2020$

Data de aceite: $11 / 03 / 2021$

\section{Como citar esse artigo:}

PERES, A.L. et al. Cuidados de enfermagem ao recém-nascido nos distintos cenários: revisão integrativa. Advances in Nursing and Health, v. 3, p. 31-47, Londrina, 2021. 


\section{INTRODUÇÃO}

A Organização Mundial de Saúde (OMS) preconiza a classificação do recémnascido conforme a idade gestacional ao nascer: a termo são os nascidos a partir de 37 até 41 semanas; o pós-termo é aquele com idade gestacional ao nascimento igual ou superior a 42 semanas. Nesse cenário, destacam-se os nascimentos prematuros, anteriores a 37 semanas, que são subclassificados em prematuros extremo (menores de 28 semanas), muito prematuro (de 28 a 31 semanas e 6 dias) e os prematuros moderados (após 32 semanas de idade gestacional) (1).

A assistência humanizada no âmbito da neonatologia não está centralizada apenas no recém-nascido, mas também em sua família. Para população neonatal, os cuidados centrados na família são essenciais para a garantia da qualidade assistencial, do menor tempo de internamento, da redução da morbimortalidade, bem como para a melhoria no prognóstico (2).

$$
\text { A equipe de Enfermagem na }
$$
abordagem à saúde neonatal desenvolve atenção humanizada quando propõe intervenções centradas na família, com vistas à conquista da integralidade do cuidado. O enfermeiro, por sua vez, assume o compromisso de cuidar dos recémnascidos, priorizando atitudes de zelo e segurança que partam de si e de sua equipe, nos diversos cenários de atenção focados na assistência (3).

Nesse sentido, conforme a Portaria no 1.459, de 24 de junho de 2011, que institui a Rede Cegonha no âmbito do SUS, os cenários de atenção à saúde pública que compõem a assistência ao recém-nascido, a princípio, são a sala de parto, onde o binômio mãe-bebê são atendidos durante o parto e o nascimento, e o alojamento conjunto, para o qual o recém-nascido é encaminhado após nascimento com boa vitalidade e permanece em companhia de sua mãe até o momento da alta hospitalar. Na vigência de alguma intercorrência, avalia-se a recomendação de internamento em uma Unidade Neonatal, para que receba os cuidados semicríticos ou intensivos.

A primeira etapa do Método Canguru é representada pela Unidade Neonatal, na qual é ofertada assistência de alta complexidade a pacientes neonatais em condições graves ou com risco para a morte. Nesse cenário, deve-se contar com uma equipe multiprofissional para acolher e 


\section{tin}

assistir os recém-nascidos, bem como os seus familiares sempre que necessário, além do aporte tecnológico de ponta para realizar diagnóstico e tratamento necessários à manutenção da vida (4-5).

Contudo, frente às situações de complexidade intermediária, os recémnascidos são transferidos para uma Unidade de Cuidado Intermediário Convencional (UCINCo). Nesse cenário de atenção, são recebidos os recém-nascidos com alta ou encaminhamento da internação em UTIN que necessitam de observação após 72 horas (4).

Já a segunda etapa do Método Canguru consiste na assistência realizada também em unidade intermediária, a Unidade de Cuidado Intermediário Canguru (UCINCa), que atende pacientes provenientes tanto da UCINCo quanto da UTIN, após melhora clínica. Nela, é realizado com contato pele a pele, bem como o preparo da família para cuidar do recémnascido com baixo peso ao nascer diante da alta hospitalar. Assim, são promovidos a formação do vínculo entre o recém-nascido e sua família, o aleitamento materno e a capacitação da família e/ou responsável para o cuidado em domicílio ${ }^{(5)}$.
O seguimento ambulatorial consiste na terceira etapa proposta pelo Método Canguru, que ocorre após a alta hospitalar, podendo estar vinculado a um ambulatório de alto risco ou à Atenção Primária em Saúde (APS). Todos os cenários de atenção visam a humanização da assistência, por meio do cuidado centrado na família ${ }^{(5)}$, o qual consiste em uma filosofia de cuidado que preza pela família como a principal unidade de cuidado

$\mathrm{Na}$ Europa, a humanização do cuidado neonatal é baseada, fundamentalmente, nos direitos do recémnascido, com projetos como a "Iniciativa Cuidados de Saúde Amigos da Criança", que foi criado no Reino Unido, em colaboração com a United Nations International Children's Emergency Fund (UNICEF) e a Organização Mundial da Saúde (OMS), em 2000. Trata-se de uma iniciativa que visa a redução do sofrimento do recém-nascido e de suas famílias, através da prática de 12 padrões, como, por exemplo, os serviços projetados especialmente para os recém-nascidos e suas famílias, empoderamento destas sobre a assistência que será prestada, entre outros (2). Nos Estados Unidos, a humanização 


\section{tin}

da assistência neonatal é centrada no cuidado integral a família, promovida com as chamadas "Family-Centered Rounds", rodas realizadas à beira leito. Estas têm como objetivo a construção, pela equipe interdisciplinar e a família, de um plano para manejo do neonato ${ }^{(2)}$.

Em países em desenvolvimento, com disparidades sociais, a humanização do cuidado é implementada mediante programas governamentais. No caso do Brasil, citam-se o Programa Nacional de Humanização da Assistência Hospitalar (PNHAH) e a Política Nacional de Humanização - o HumanizaSUS. Tais programas governamentais visam a melhoria dos cuidados em saúde em geral e consideram as questões de direitos da criança, adolescente e família, que figuram na Constituição Federal e no Estatuto da Criança e do Adolescente (ECA) (6). O ECA, em seu Art. 70, apresenta o direito da criança à saúde e à proteção da vida, mediante políticas públicas que permitam o nascimento e o desenvolvimento sadio (7).

Frente às crescentes necessidades de internamentos, tendo em vista a atenção qualificada à saúde dos neonatos, é necessário fundamentar as estratégias, com respeito à singularidade do recém-nascido, aplicando-se o cuidado centrado na família, o que se torna imprescindível para o aprimoramento da assistência de enfermagem. Essas estratégias possibilitam a qualificação dos profissionais, dos serviços e sistemas de saúde, com impacto positivo na redução da morbimortalidade na primeira infância. Assim, conclui-se que a síntese de evidências científicas possa fortalecer e fundamentar as intervenções de enfermagem aos dos recém-nascidos, nos diferentes cenários de assistência à saúde. Portanto, o objetivo deste estudo foi identificar os cuidados de enfermagem ao recém-nascido, nos diversos cenários de atenção.

\section{MÉTODO}

Trata-se de revisão integrativa com base no referencial metodológico de Ganong (1887), nas etapas preconizadas: 1) Elaboração da pergunta de pesquisa; 2) Amostragem; 3) Representação das características dos estudos primários; 4) Análise dos achados; 5) Interpretação dos resultados; e 6) Apresentação da revisão (8). Também foram aplicadas as recomendações 


\section{tin}

do Preferred Reporting Items for Systematic Reviews and Meta-Analyses (PRISMA) (9).

A princípio, estruturou-se o problema de pesquisa a partir da seguinte questão: Quais os cuidados de enfermagem neonatais realizados nos diferentes cenários de atenção? Esta pergunta de pesquisa foi desenvolvida segundo o acrônimo PICo: P (população) - os recém-nascidos; I (intervenção) - os cuidados de enfermagem; Co (contexto) - nos diversos cenários da atenção (10).

A coleta de dados ocorreu no período de janeiro a março de 2018, com a estratégia de busca composta pelos descritores "enfermagem neonatal" ("neonatal nursing"), "cuidados de enfermagem" ("nursing care") e "recémnascido"("infant"), intercalados com o operador booleano $A N D$, nas bases de dados US National Library of Medicine (Pubmed), SciVerse Scopus, Medical Literature Analysis and Retrieval System Online (Medline), Cumulative Index to Nursing and Allied Health Literature (CINAHL) e Web of Science. Os descritores foram obtidos do Medical Subject Headings (MeSH).

Os critérios para a inclusão dos estudos primários foram os seguintes: artigos em inglês, português e espanhol, focados na população de recém-nascidos e publicados entre 2015 a 2017. Optou-se por esse recorte temporal devido ao intuito de aproximação do conhecimento mais atual, aplicado aos cuidados de enfermagem aos recém-nascidos e que respondiam à pergunta de pesquisa. Como critérios de exclusão, foram considerados os seguintes fatores: editoriais de revistas, cartas ao editor, resumos em anais, revisões e estudos que abordavam doenças neonatais específicas com aprofundamento em outras categorias profissionais.

Os artigos foram selecionados por dois revisores independentes e, durante o processo de seleção, não houve discordância entre eles, não sendo necessária a inclusão de um terceiro revisor. Na sequência, para análise criteriosa da qualidade metodológica dos estudos selecionados, foi aplicado o instrumento proposto por Downs e Black (1998), denominado de Cheklist for Measuring Study Quality (11). Este é composto por 27 itens, dos quais oito não foram aplicados aos estudos selecionados por avaliarem a qualidade metodológica de estudos experimentais, que não constam da 
seleção. Assim, aplicaram-se 19 itens para análise, sendo que as respostas registradas às perguntas do checklist foram 'sim', 'não' e 'não consegue determinar'.

Os estudos primários incluídos na amostra desta revisão integrativa apresentaram pontuação acima de 14 pontos, ou seja, 70\% do checklist foi respondido positivamente, com 'sim'. A aplicação do instrumento (11) na última etapa de seleção dos artigos propiciou a exclusão de seis estudos que não tinham qualidade metodológica satisfatória. 0 processo detalhado da seleção dos estudos primários está apresentado na Figura 1.

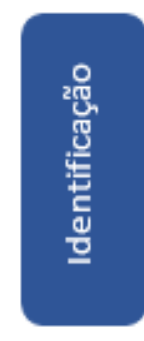

Artigos identificados em bases de dados: SCOPUS ( $n=243) ;$ Web of Science $(n=24)$; PUBMED $(n=207) ;$ MEDLINE $(n=363)$; CINAHL $(n=192)$ Total $(n=1029)$

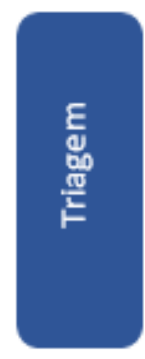

Artigos restantes após remoção de duplicados $(n=995)$
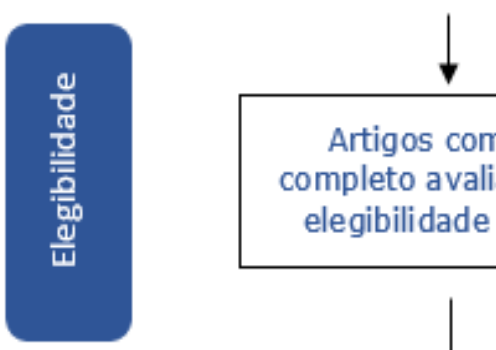

Artigos com texto completo avaliados para elegibilidade $(n=60)$
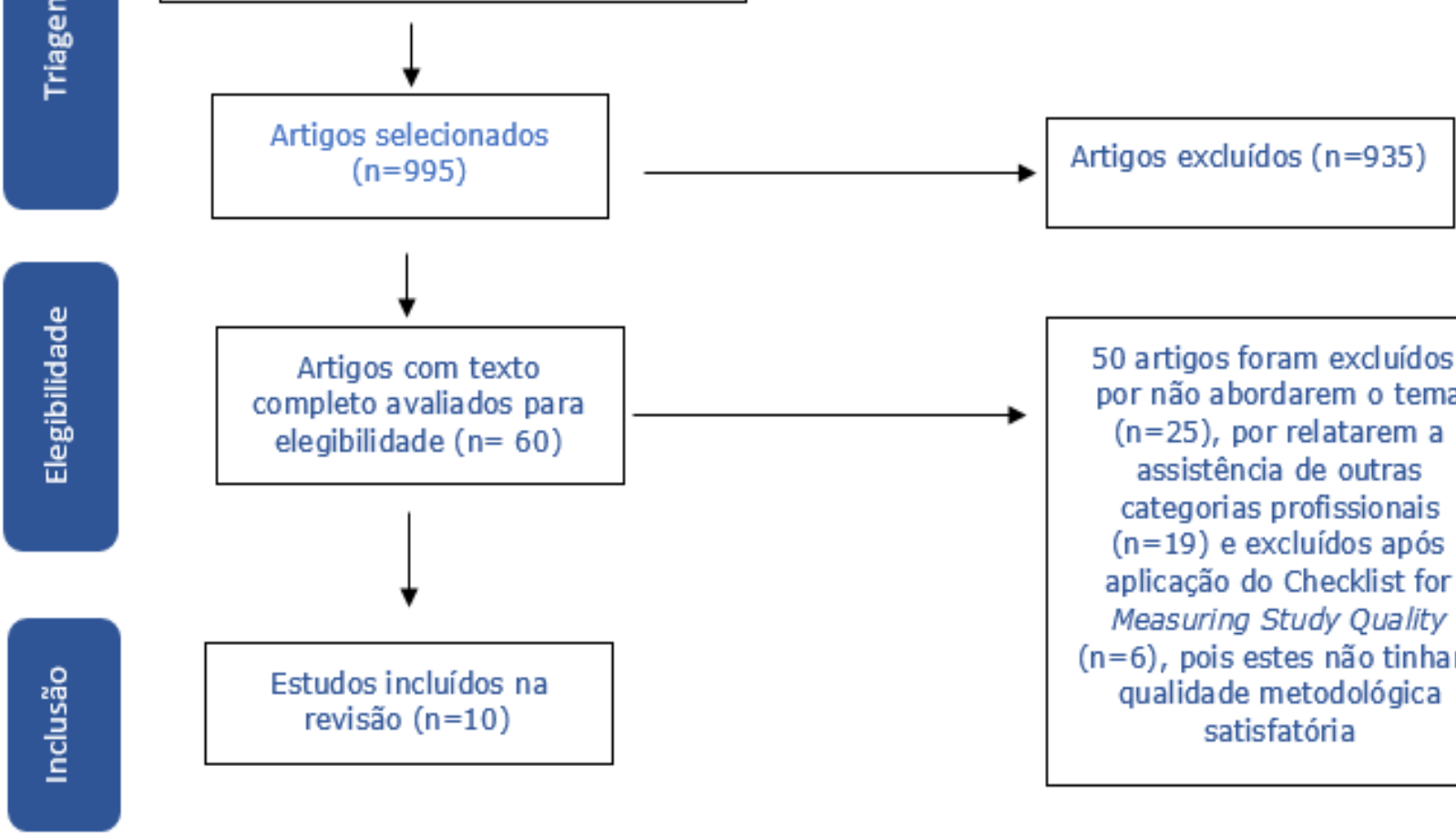

Figura 1 - Fluxograma de seleção dos estudos primários incluídos na amostra desta revisão integrativa, segundo as recomendações do PRISMA (9). Paraná, Brasil, 2018. 


\section{RESULTADOS}

A amostra desta revisão integrativa

foi composta por 10 estudos primários, dos quais 4 (13-16) foram publicadas em 2017, 2 (17-18) em 2016 e $4^{(19-22)}$ em 2015. O Brasil apresentou o maior número de publicações, totalizando $6(13,17-21)$, enquanto os demais estudos foram publicados nos seguintes países: Estados Unidos (22), Holanda (14), Itália (15) e Indonésia (16). Em relação ao idioma de publicação, foram identificados 5 estudos em português (13,16-21) e 5 na língua inglesa (13-17,22). Os autores de todos os artigos analisados eram enfermeiros (13-22), os quais desenvolvem suas atividades em escolas de nível superior, departamentos e hospitais.

Quanto aos delineamentos das pesquisas, 7 estudos (13-17,21,22) apresentaram abordagem quantitativa e $3(18,20,21)$ qualitativa. No Quadro 1, são apresentadas as características de cada estudo primário segundo desenho do estudo, nível de evidência, participantes, cenário de atenção, cuidado de enfermagem e principais resultados. Cada estudo foi identificado por uma letra, seguida de sua referência.

\section{Quadro 1 - Caracterização dos estudos primários incluídos na amostra desta revisão integrativa. Paraná, Brasil, 2018.}

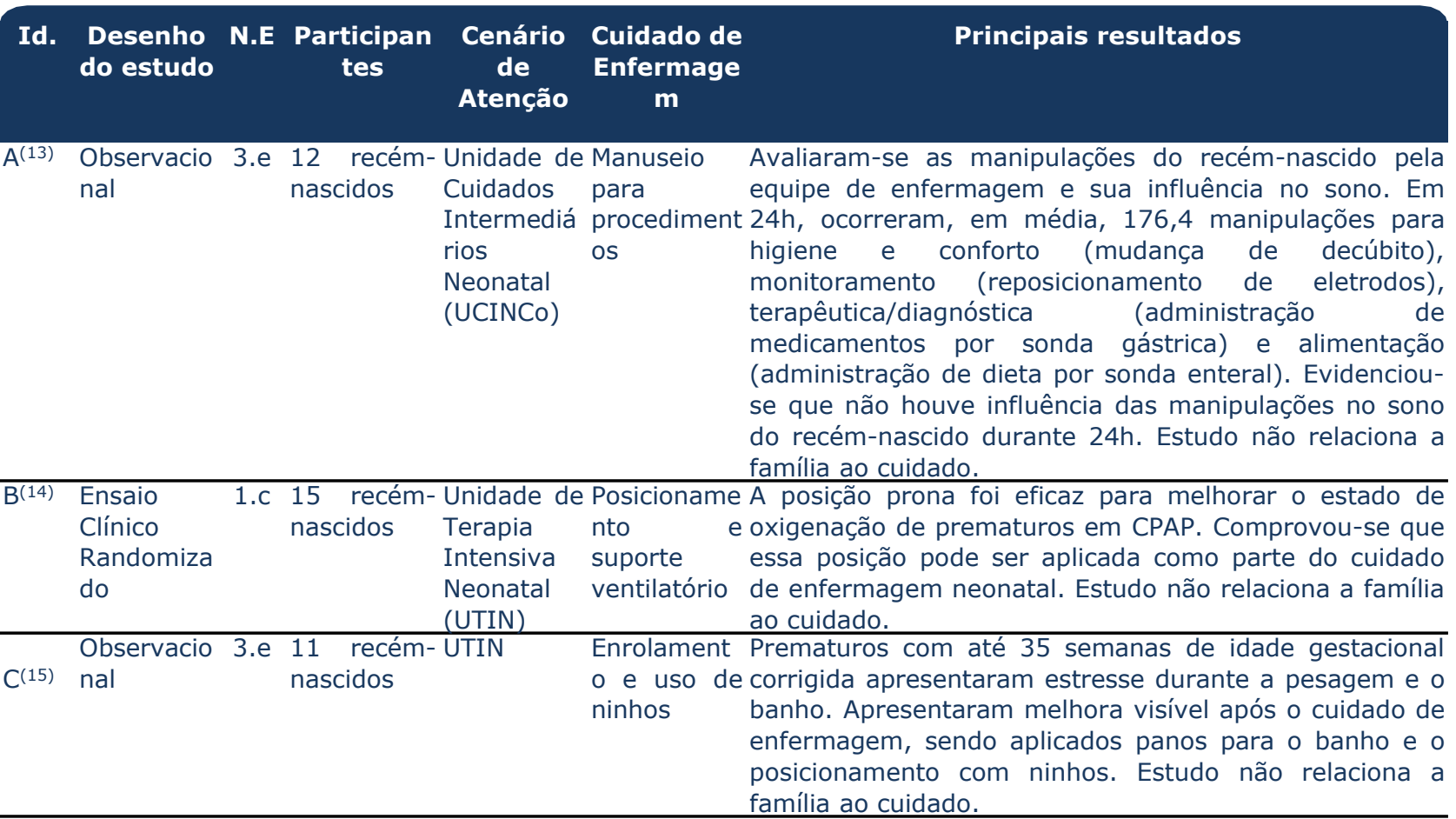


A atuação da enfermagem nas boas práticas de cuidado ao recém-nascido foi foco dos resultados de todos os estudos primários desta revisão integrativa, os quais relacionam a importância fundamental da atuação da enfermagem, qualificada na assistência neonatal, como parte da equipe multiprofissional. Apesar de a maioria dos

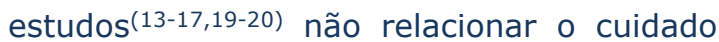
realizado com abrangência familiar, compreendeu-se, nas suas discussões, que os cenários nos quais foram realizados aplicam a filosofia do cuidado centrado na família, uma vez que fazem inserção desta na assistência.

\section{DISCUSSÃO}

Dentre os principais cuidados de enfermagem ao recém-nascido discutidos pela literatura científica selecionada, foram observados o manuseio mínimo(13,17,19,22), o posicionamento(14,17,21), a qualidade do suporte ventilatório(14,16), o uso de panos e ninho para banho e pesagem ${ }^{(15)}$, a prevenção de leões de pele(20), bem como todo o contexto do cuidado relacionado ao conforto, à proteção e ao bem-estar(18). Proporcionar um sono de qualidade mediante práticas de sono seguro, como a posição supina, a utilização do berço plano sem inclinação e ninho, sem dispositivos de posicionamento ou brinquedos ou cobertores, viabiliza a regulação da homeostase e estimula o neurodesenvolvimento. $\mathrm{O}$ ciclo de sono qualifica o tratamento e o prognóstico dos recém-nascidos, prevenindo complicações neurológicas $(23,24)$

Dessa forma, preocupar-se em tornar - ambiente das UTIN propício para a preservação do sono através das práticas de

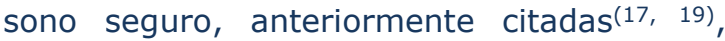
pode ser considerado um relevante cuidado de enfermagem a longo prazo. Os membros da equipe de enfermagem apresentam-se como os principais atores das intervenções assistenciais realizadas para a manutenção da vida dos recém-nascidos(13). A conduta do enfermeiro pode influenciar toda a equipe multiprofissional, como também as famílias desses recém-nascidos para darem continuidade em ambiente domiciliar(22).

A posição ventral, também conhecida como prona, contribui para a redução do despertar, proporcionando maior tempo de sono (17). Além de contribuir com a 


\section{tin}

qualidade do sono, esse cuidado também proporciona melhora da digestão por dieta via enteral, reduz o estresse e a dor (24), melhora a oxigenação, sobretudo dos recémnascidos prematuros que utilizam ventilação não invasiva(17). Coloca-se como uma prática a ser utilizada nos diversos cenários da atenção ao recém-nascido, sendo introjetada de forma rotineira na assistência de enfermagem. Entretanto, seus efeitos adversos precisam ser considerados, tendo em vista a possibilidade de diminuição da oxigenação cerebral e o aumento do risco para Síndrome da Morte Súbita(24).

Em relação ao suporte ventilatório, além da preocupação com o posicionamento adequado(14) $^{(14}$ para a melhora da ventilação pulmonar, também se discute a importância da implementação de diretrizes para controle da oferta de oxigênio(16), a fim de reduzir quadros de hipoxemia. Destaca-se que os recém-nascidos prematuros e com baixo peso ao nascer necessitam de suporte ventilatório por tempo prolongado, devido à imaturidade pulmonar, sendo que o oxigênio ofertado promove estresse respiratório que resulta em patologias pulmonares(16-22, 25), como a Displasia Broncopulmonar.

O banho e a pesagem são cuidados de enfermagem que estão presentes nos diversos cenários de atenção ao recémnascido. Quando realizado nas primeiras 24 horas de vida, podem provocar estresse neonatal(15), devido às alterações térmicas e cardiorrespiratórias identificadas pela redução da oxigenação. O banho é um processo cultural, que tem como benefícios a limpeza corporal, o estímulo da circulação sanguínea da pele e tecido subcutâneo, além do conforto e bem-estar(26). Sua realização, respeitando os critérios clínicos do recémnascido, justifica um modelo de assistência humanizado.

$\mathrm{Na}$ prevenção dos efeitos adversos ocasionados em relação ao banho, considerase a implementação de outras formas de higienização da pele, como o uso de panos úmidos e intervalos de tempo maiores (27). Igualmente, durante o procedimento de pesagem, deve-se atentar para os seguintes procedimentos: a manipulação mínima relacionada ao quadro clínico do recémnascido; o enrolamento antes de colocá-lo na balança, para diminuição do estresse térmico e preservação da proteção neurológica; o uso de balança digital para aumento da rapidez e confiabilidade do procedimento; e o apoio da cabeça do recém-nascido com uma das mãos 
do profissional durante sua mobilização(28).

O estresse afeta de forma permanente os sistemas neurobiológicos, fisiológicos e hormonais do recém-nascido (29), estando também relacionado à dor. Por isso, a equipe de enfermagem precisa ser sensibilizada e capacitada para o uso das medidas não farmacológicas para o alívio da dor e o controle do estresse, como a administração de glicose via oral antes da realização de procedimentos doloroso, a oferta de sucção não-nutritiva, o contato pele a pele, o uso de enrolamento, por meio de malha tubular e cueiros, e a contenção facilitada(30), que representam a humanização nos cuidados ofertados ao recém-nascido.

A enfermagem também atua no cuidado com a pele e implementa cuidados para prevenção de lesões, no momento do banho: a hidratação da pele, o controle térmico e o manuseio das fixações de dispositivos invasivos(20). A pele do recémnascido é imatura, delgada e permeável, além de apresentar maior extensão de superfície corporal em relação ao seu peso. Produz menor quantidade de suor e possui menor quantidade de tecido adiposo, tornando-os suscetíveis à perda de calor. $\mathrm{Na}$ pele do recém-nascido prematuro, há uma diminuição dos eletrólitos, água, proteínas e, primordialmente, de calor(31).

Estudo realizado no Egito aponta que as lesões cutâneas estavam presentes em 76\% dos recém-nascidos internados em uma UTIN, sendo que, destas, $41 \%$ eram lesões transitórias e benignas, como a miliária e as manchas de nascença. Outro dado importante identificado é que as infecções fúngicas, como a candidíase, ocorrem devido à associação entre a imaturidade da pele e a do sistema imunológico. Prevenir lesões e infecções contribui significativamente para a melhora do prognóstico geral do recémnascido(32).

O controle térmico do recém-nascido prematuro é um desafio. A redução da temperatura corporal acontece devido a desiquilíbrio entre aumento da perda de calor, não compensada por sua limitada produção. Os cuidados de enfermagem estão relacionados com a prevenção de perda de calor, pela aplicação de técnicas simples como o envolvimento do corpo e da cabeça do recém-nascido em plástico logo após o nascimento, o uso de incubadoras e berços aquecidos, a utilização de colchões 


\section{H}

exotérmicos e o contato pele a pele(33).

Ademais, a promoção do aleitamento materno também é um cuidado de enfermagem, não discutido nos estudos primários em análise. Ele proporciona o ganho de peso em recém-nascidos prematuros e de baixo peso, promove 0 vínculo, conforto e alívio da dor, crescimento, desenvolvimento e neurodesenvolvimento adequados, além de aumento da sobrevida com melhora do prognóstico e diminuição das intercorrências e da morbimortalidade(34).

Embora os cuidados especializados prestados ao recém-nascido, sobretudo aos prematuros, envolvam os procedimentos simples, bem como os complexos, a equipe multiprofissional deverá estar alerta para proporcionar a interação do recém-nascido com sua família, a fim de fortalecer o vínculo entre eles, respeitando-o, assim, como sujeito e não como objeto de trabalho( ${ }^{(34)}$. 0 acolhimento com informações claras sobre o estado de saúde e os direitos e deveres da família contribui para o cuidado centrado na família, estabelecendo a comunicação efetiva, a confiança e o respeito, os quais se caracterizam como aspectos necessários para uma relação democrática que viabiliza a tomada de decisão baseada em evidências(35).

Demonstrou-se que os estudos primários expressam preocupação com qualificação dos profissionais de saúde que atuam em UTIN para a realização de cuidados para o conforto e a proteção, em especial aos recém-nascidos prematuros. A ausência de discussão atualizada quanto aos cuidados com amamentação, vacinação, controle da dor, sobre cuidados de enfermagem em distintos cenários de atenção, como o alojamento conjunto e a sala de parto, foi considerada como uma limitação desta revisão integrativa, tendo em vista a relevância dos aspectos de cuidados e dos cenários adequados para a promoção do cuidado ao recém-nascido e família.

\section{CONCLUSÃO}

Portanto, a aplicação de cuidados de enfermagem ao recém-nascido com foco na prática segura do sono, melhora do suporte ventilatório, manuseio mínimo, redução dos estímulos sensoriais e ambientais, uso de ninhos e panos para redução do estresse no banho e pesagem, atenção à hidratação da 


\section{tin}

pele e regulação da temperatura para prevenção de lesões destacou-se na redução dos eventos adversos ocasionados pelas intervenções. Além disso, essa aplicação visa a manutenção da sobrevida dos recémnascidos, sobretudo os prematuros e os nascidos com baixo peso.

Por conseguinte, a aplicação das evidências científicas identificadas acerca dos cuidados de enfermagem nos distintos cenários de atenção ao recém-nascido contribui para o desenvolvimento da enfermagem como profissão. Assim, foi possível evidenciar que os estudos que compõem esta revisão contribuem para a assistência em enfermagem ao recémnascido com enfoque no cuidado individualizado e centrado na família, refletindo em melhores condições de saúde pública por promover a redução dos indicadores de mortalidade no período neonatal, sobretudo no neonatal precoce. Além disso, impactará positivamente na redução da morbidade e na qualidade de vida dessas crianças, transformando a realidade de saúde da comunidade.

Sugere-se a realização de novos estudos a fim de proporcionar a qualificação da prática assistencial no cenário da atenção neonatal, em outros cenários, como nas UCINCo, pois é um serviço preconizado por políticas públicas de saúde que seguem diretrizes para um modelo de assistência neonatal humanizado. Além disso, sugeremse investigações no Alojamento Conjunto e na Atenção Primária à Saúde, devido à ênfase recomendada ao cuidado oferecido ser na promoção da saúde e prevenção dos agravos em recém-nascidos.

\section{REFERÊNCIAS}

1. World Health Organization. Born too Soon. The Global Action Report on Preterm Birth. Geneva: WHO; 2012

2. Tripodi, M., Siano, M. A., Mandato, C., De Anseris, A. G. E., Quitadamo, P., Guercio Nuzio, S., Vajro, P. Humanization of pediatric care in the world: focus and review of existing models and measurement tools. Italian Journal of Pediatrics [Internet] 2017;43(1). [acesso em: 26 de abr. 2020] Disponivel: https://doi.org/10.1186/s13052017-0394-4

3. Costa JVS, Sanfelice CFO, Carmona EV. Humanização da assistência neonatal na ótica dos profissionais de enfermagem. Rev enferm UFPE [Internet] 2019;13(242) [acesso em: 26 de abr. 2020] Disponivel: https://doi.org/10.5205/19818963.2019 .242642 


\section{tin}

4. Ministério da Saúde (BR). Portaria no 1.459, de 24 de junho de 2011, institui a Rede Cegonha no âmbito do SUS. Diário Oficial da União, [Internet] 24 jun 2011 [acesso em 06 mar 2021]. Disponível: https://bvsms.saude.gov.br/bvs/saudelegis/g m/2011/prt1459_24_06_2011.html

5. Ministério da Saúde (BR). Portaria $n^{\circ}$ 1.683, de 12 de julho de 2007, institui Normas de Orientação para a Implantação do Método Canguru. Diário Oficial da União, [Internet] 12 jul 2007 [acesso em 06 mar 2021].

Disponível:

https://bvsms.saude.gov.br/bvs/saudelegis/g m/2007/prt1683_12_07_2007.html

6. Noda LM, Alves MVMFF, Gonçalves MF, Silva FS, Fusco SFB, Avila MAG. Humanization in the Neonatal Intensive Care Unit from parents' perspective. REME - Rev Min Enferm. [Internet] 2018:22(1078) [acesso em: 27 ago. 2020]. Disponivel: http://www.dx.doi.org/10.5935/1415-

2762.20180008

7. Brasil. Lei Federal n. 8069, de 13 de julho de 1990. Dispõe sobre o Estatuto da Criança e do Adolescente e dá outras providências. Diário Oficial da República Federativa do Brasil, Brasília, 13 jul. 1990. Seção 1:1.

8. Ganong L. Integrative reviews of nursing research. Res Nurs Health. 1987 ;10(1) [acesso em: 17 abr 2018] Disponivel: https://doi.org/10.1002/nur.4770100103.

9. Galvão TF, Pansani TSA, Harrad D.
Principais itens para relatar Revisões sistemáticas e Meta-análises: A recomendação PRISMA. Epidemiol. Serv. Saúde [Internet] 2015;24(2) [acesso em: 23 mar 2018] Disponível: http://dx.doi.org/10.5123/S167949742015000200017

10. Apóstolo JLA. Síntese da evidência no contexto da translação da ciência. Coimbra, Portugal: Escola Superior de Enfermagem de Coimbra, 2017.

11. Downs SH, Black N. The feasibility of creating a checklist for the assessment of the methodological quality both of randomised and nonrandomised studies of health care interventions. J Epidemiol Community Health [Internet] 1998;52 (6) [acesso em: $17 \mathrm{abr}$ 2018]

Disponível: http://dx.doi.org/10.1136/jech.52.6.377. PMid: 9764259.

12. Joanna Briggs Institute [Internet] Adelaide: The University of Adelaide; [acesso em 06 mar 2021]. The JBI Model of Evidence-Based Healthcare; [3 telas]. Disponivel: https://jbi.global/jbi-approachto-EBHC

13. Maki MT, Orsi KCSC, Tsunemi MH, Hallinan MP, Pinheiro EM, Avelar AFM. O efeito da manipulação sobre o sono do recém-nascido prematuro. Acta paul. enferm. [Internet]. 2017;30(5) [acesso em: 22 mar 2018] Disponível: http://dx.doi.org/10.1590/19820194201700071.

14. Utario Y, Rustina Y, Waluyanti FT. The 
Quarter Prone Position Increases Oxygen Saturation in Premature Infants Using Continuous Positive Airway Pressure. Compr Child Adolesc Nurs [Internet]. 2017;40(sup1) [acesso em: 18 abr 2018]. Disponível: http://dx.doi.org/10.1080/24694193.2017.1 386976

15. Bembich S, Fiani G, Strajn T, Sanesi C, Demarini S, Sanson G. Longitudinal Responses to Weighing and Bathing Procedures in Preterm Infants. J Perinat Neonatal Nurs [Internet] 2017;31(1) [acesso em: 18 abr 2018] Disponível: http://dx.doi.org/10.1097/jpn.00000000000 00228]

16. Van Zanten HA, Pauws SC, Beks EC, Stenson BJ, Lopriore E, Te Pas ABVAN. Improving manual oxygen titration in preterm infants by training and guideline implementation. Eur J Pediatr [Internet] 2017;176(1) [acesso em: $18 \mathrm{abr}$ 2018] Disponível em: http://dx.doi.org/10.1007/s00431-0162811-x

17. Modesto IF, Avelar AF, Pedreira L, Pradella-Hallinan M, Avena MJ, Pinheiro EM. Effect of sleeping position on arousals from sleep in preterm infants. J Spec Pediatr Nurs [Internet] 2016;21(3) [acesso em: 17 abr 2018] Disponível: http://dx.doi.org/10.1111/jspn.12147

18. Ferreira JHP, Amaral JJF, Lopes MMCO. Nursing team and promotion of humanized care in a neonatal unit. Rev Rene [Internet]
2016;17(6) [Acesso em: 18 abr 2018] Disponível:http://dx.doi.org/10.15253/21756783.2016000600003

19. Orsi KCSC, Llaguno NS, Avelar AFM, Tsunemi MH, Pedreira MLG, Sato $M H$, et al. Effect of reducing sensory and environmental stimuli during hospitalized premature infant sleep. Rev. esc. enferm. [Internet] 2015;49(4) [acesso em: 17 abr 2018] Disponível:http://dx.doi.org/10.1590/s0080623420150000400003

20. Santos SV, Costa R. Prevenção de lesões de pele em recém-nascidos: o conhecimento da equipe de enfermagem. Texto contexto enferm. [Internet]. 2015;24(3) [acesso em: 18 abr 2018] Disponível: http://dx.doi.org/10.1590/0104-

0707201501123_014

21. Toso BRGO, Viera CS, Valter JM, Delatore S, Barreto GMS. Validação de protocolo de posicionamento de recém-nascido em Unidade de Terapia Intensiva. Rev. Bras. Enferm. [Internet] 2015;68(6) [acesso em: 18 abr 2018] Disponível:

http://dx.doi.org/10.1590/0034-

$7167.2015680621 \mathrm{i}$

22. Hwang SS, O'Sullivan A, Fitzgerald E, Melvin P, Gorman T, Fiascone JM. Implementation of safe sleep practices in the neonatal intensive care unit. J Perinatol [Internet] 2015;35(10) [acesso em: $18 \mathrm{abr}$ 2018] Disponível: http://dx.doi.org/10.1038/jp.2015.79 


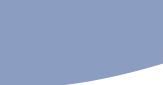

23. Llaguno NS, Pedreira MLG, Avelar AFM, Avena MJ, Tsunemi MH, Pinheiro EM. Avaliação polissonográfica do sono e vigília de recém-nascidos prematuros. Rev Bras Enferm [Internet] 2015;68(6) [acesso em: 18 abr 2018] Disponível: http://dx.doi.org/10.1590/0034-

7167.2015680616i

24. Ma M, Noori S, Maarek JM, Holschneider DP, Rubinstein EH, Seri I. Prone positioning decreases cardiac output and increases systemic vascular resistance in neonates. J Perinatol [Internet] 2015;35(6) [acesso em: 18 abr 2018] Disponível: http://dx.doi.org/10.1038/jp.2014.230

25. Lissauer T, Duke T, Mellor K, Molyneux L. Nasal CPAP for neonatal respiratory support in low and middle-income countries. Arch Dis Child Fetal Neonatal Ed. [Internet] 2017;102(3) [acesso em: 18 abr 2018]

Disponível: http://dx.doi.org/10.1136/archdischild-2016311653

26. Filho GGF, Passos JOS, Almeida VA, Ribeiro CMA, Souza JC, Silva GFA, et al. Thermal and cardiorespiratory newborn adaptations during hot tub bath. Int Arch of Med [Internet] 2017;10(85) [acesso em: 18 abr 2018] Disponível: http://dx.doi.org/10.3823/2355

27. Ruschel LM, Pedrini DB, Cunha MLC. Hypothermia and the newborn's bath in the first hours of life. Rev Gaúcha Enferm.
[Internet] 2018;39 [acesso em 01 jun 2019]. Disponível: https://doi. org/10.1590/19831447.2018.20170263.

28. Gomes T, Sant'ana A, Neto M, Porto F. Fundamentals of care in weighing the newborn / Fundamentos do cuidado na pesagem do recém-nascido. Revista de Pesquisa: Cuidado é Fundamental Online [Internet]. 2019 Jan 1; [acesso em 2019 Jul 3]; 11(1): 74-79. Disponível: http://dx.doi.org/10.9789/21755361.2019.v11i1.74-79.

29. Brummelte S, Chau CM, Cepeda IL, Degenhardt A, Weinberg J, Synnes AR et al. Cortisol levels in former preterm children at school age are predicted by neonatal procedural pain-related stress. Psychoneuroendocrinology[Internet] 2 015;51 [acesso em: 18 abr 2018] Disponível:

http://dx.doi.org/10.1016/j.psyneuen.2014.0 9.018

30. Motta GCP, Cunha MLC. Prevention and non-pharmacological management of pain in newborns. Rev. Bras. Enferm. [Internet] 2015; 68(1) [acesso em 04 jun 2019]. Disponível: http://dx.doi.org/10.1590/00347167.2015680118p

31. Haveri FTTS, Inamadar AC. A CrossSectional Prospective Study of Cutaneous Lesions in Newborn. ISRN Dermatol [Internet] 2014; 20(2014) [acesso em: 18 abr 2018]

Disponível:http://dx.doi.org/10.1155/2014/3 60590 
32. Shehab MM, Youssef DM, Khalil MM. Prevalence of cutaneous skin lesions in neonatal intensive care unit: A single center study. J Clin Neonatol. [Internet] 2015; 4(3) [acesso em 01 jun 2019]. Disponível: http://dx.doi.org/10.4103/2249-

4847.159872

33. Wilson E, Maier RF, Norman M, Misselwitz B, Howell EA, Zeitlin J. Admission Hypothermia in Very Preterm Infants and Neonatal Mortality and Morbidity. J Pediatr. [Internet] 2016; 175 [acesso em 31 mai 2019].

Disponível: http://dx.doi.org/10.1016/j.jpeds.2016.04.0 16
34. Lopes TRG, Oliveira SS, Pereira IRRBO, Romeiro IMM, Carvalho JBL. Humanization of care to newborns in the kangaroo method: experience report. J Nurs UFPE online. [Internet] 2017; 11(11) [acesso em 02 jun 2019].

Disponível:

https://doi.org/10.5205/1981-8963v11i11a25089p4492-4497-20

35. Corrêa AR, Andrade AC, Manzo BF, Couto $D L$, Duarte ED. The family-centered care practices in newborn unit nursing perspective. Esc. Anna Nery [Internet]. 2015; 19(4) [acesso em 06 mar 2021] Disponível: https://doi.org/10.5935/14148145.20150084 